\title{
A NEW LOOK AT BEHÇET'S DISEASE: CONSTRICTIVE PERICARDITIS
}

Adriana Guimarães de Farias ${ }^{1, *} ;$ Mariza Marques de Oliveira ${ }^{1}$

1. Hospital de Base Dr Ary Pinheiro, Porto Velho (RO), Brazil.

*Corresponding author: adrianagdefarias@gmail.com

\section{BACKGROUND}

Behçet's disease is a rare but severely debilitating vasculitis that typically manifests as a mucocutaneous disease, with orogenital ulcers and skin lesions; however, involvement of the musculoskeletal system, eye, nervous system, gastrointestinal tract, vascular manifestations, urogenital tract and cardiopulmonary system can lead to significant morbidity and mortality.

\section{CASE REPORT}

Patient A.B.S., female, 42 years old, hospitalized in 2009 presenting dyspnea on small efforts, edema of the lower limbs and asthenia. Chest computed tomography showed intense calcification of the pericardium and possible mass that compressed the right ventricle and invaded the interventricular septum. She underwent pericardiectomy and the anatomopathological examination showed a nonspecific inflammatory process. In 2017, she sought a rheumatologist for worsening heart failure and polyarthralgia, initially with a history of three abortions. She reported recurrent oral ulcers. She was submitted to electroneuromyography, which showed polyneuropathy, evolving with multiplex mononeuritis. Positive HLA-B51 determination. Neuritis observed by an ophthalmologist and thrombosis of the right external jugular vein. With a diagnosis of Behçet's disease established, pulse therapy with methylprednisolone and cyclophosphamide, human immunoglobulin and azathioprine was initiated, without success. Started rituximab in January 2019, with good response.

\section{CONCLUSION}

Behçet's disease should be included in the differential diagnoses of young patients with severe heart failure without a defined cause. 